\title{
Numerical Study of a Plane Poiseuille Channel Flow of a Dielectric Liquid Subjected to Unipolar Injection.
}

\author{
Philippe Traoré, Jian Wu, Christophe Louste \\ Institut PPRIME, Département Fluide-Thermique-Combustion, \\ Boulevard Pierre et Marie Curie, BP 30179, \\ 86962 Futuroscope-Chasseneuil, France \\ Pedro A. Vázquez \\ Departamento de Física Aplicada III \\ Universidad de Sevilla, ESI \\ Camino de los Descubrimientos s/n \\ 41092 Sevilla, Spain \\ and Alberto T. Pérez \\ Departamento de Electrónica y Electromagnetismo \\ Universidad de Sevilla, Facultad de Física \\ Avenida Reina Mercedes s/n \\ 41012 Sevilla, Spain
}

\begin{abstract}
In this paper, the interaction between a plane Poiseuille channel flow and an electroconvective movement induced by the electric field is numerically investigated. A flow is generated by an inlet parabolic profile in a rectangular duct. Space charges are injected in the flow through a metallic electrode placed on one of the channel walls and brought to a given potential. Transient numerical simulations have been carried out to investigate the structure of the flow. The entire set of the coupled Navier-Stokes and EHD equations is solved using an efficient finite volume technique. The behavior of the flow subjected to an applied voltage between the two electrodes is analyzed and time evolution of the charge density distributions is presented. The interaction between the convective movement induced by space charge injection and electric field and the mainstream flow, emphasizes the appearance of periodic transverse traveling waves convected in the channel. The dynamic of the flow through the variation of the ionic mobility parameter $M$ is investigated. For a given Reynolds number and ionoc mobility parameter $M$ it exists a threshold value $T_{c}$ of the instability parameters $T$ above which the transverse traveling rolls appear or not. When $T$ is increased, different flow regimes have been highlighted starting from a complete steady state up to a fully unsteady electro-plumes flow configuration.
\end{abstract}

Index Terms - Electroconvection, Poiseuille flow, charge injection, electroconvective rolls, electro-plumes instability, numerical simulation.

\section{INTRODUCTION}

CONSIDERABLE interest has been shown in recent year in fluid motion driven by Coulomb forces which arises in many natural situations and industrial process. The resulting flow which occurs when an electric field is applied across a dielectric liquid containing space charge have received much attention by the scientific engineering and industrial communities. In much of these studied situations the electric field is the cause of the movement of the flow itself by electroconvection. Here we are studying the effect induced by space charge injection and electric field on a plane Poiseuille flow. This kind of flow, when the applied potential difference is replaced by a temperature difference between the lower and 
upper walls and known as mixed- convection, has been widely studied over the last tricades. [1]-[5] A very comprehensive and complete review of this subject has been presented by Nicolas [3]. However when the role played by the temperature difference in mixed-convection is replaced by the applied voltage giving rise to what can be called "mixed electro-convection" only very few studies are available. Agraït [6], Castellanos [7], Lara et al [8] conducted stability analysis to determine the effect of the injection level on the stability of the flow. Martinelli et al [9] performed also a linear modal and non-modal stability analysis of this flow. The analogy between mixed thermo-convection and mixed electroconvection is very strong. This paper is devoted to the numerical analysis of a convective flow of a dielectric liquid in a horizontal channel subjected to unipolar injection.

In the following section we state the problem and its governing equations. We shall describe the numerical method used in this study. Main results will be discussed in Section 3. Finally, a conclusion is summarized in section 4 .

\section{PROBLEM FORMULATION AND NUMERICAL METHOD}

\subsection{GOVERNING EQUATIONS}

The system under consideration in this article is a channel of length $L$ and height $H$ which is filled with a dielectric liquid (see Figure 1). The fluid of density $\rho$, dynamic viscosity $\eta$ and permittivity $\varepsilon$, is assumed to be incompressible and perfectly insulating. On the left side of the domain the liquid penetrates the domain with an imposed inlet parabolic velocity profile. On the right side of the domain, the fluid can leave the domain. A difference of potential $\Delta V=V_{0}-V_{1}$ between the lower and upper walls where electrodes are placed is applied which generates a vertical electric field and charge injection. We consider the limit case of homogeneous and autonomous unipolar injection, which means that the charge injection arises from one electrode and the density of injected charges is always constant and not related to the electric field.

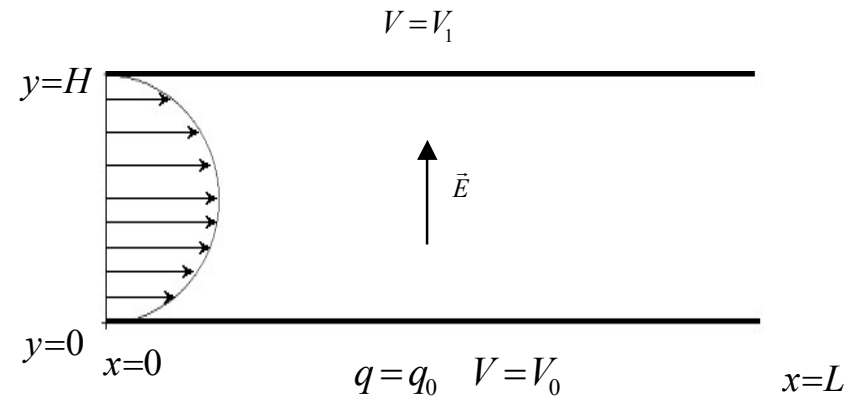

Figure 1. Sketch of the physical domain.

The problem is formulated considering the usual hypotheses of a Newtonian and incompressible fluid of dynamic viscosity $\mu$ and density $\rho$ governed by the Navier-Stokes and ElectoHydro-Dynamic (EHD) equations as follows:

$$
\begin{aligned}
& \nabla \cdot \vec{u}=0 \\
& \rho_{0}\left(\frac{\partial \vec{u}}{\partial t}+(\vec{u} \cdot \nabla) \vec{u}\right)=-\nabla \tilde{p}+\mu \Delta \vec{u}+q \vec{E} \\
& \frac{\partial q}{\partial t}+\nabla \cdot(q(\vec{u}+K \vec{E}))=0 \\
& \Delta V=-\frac{q}{\varepsilon} \\
& \vec{E}=-\nabla V
\end{aligned}
$$

where $\vec{u}$ is the velocity, $\tilde{p}$ is the modified pressure which includes the contribution from the electrostriction force term, $q$ the charge density, $K$ and $\varepsilon$ are respectively the ionic mobility and the permittivity of the liquid in consideration.

For universality in the description of such studies it is particularly convenient to work with non dimensional equations. In order to transform the last set of equations into a non dimensional one we introduce the following dimensionless quantities denoted with a star:

$x_{i}^{*}=\frac{x_{i}}{H}, \quad \rho^{*}=\frac{\rho}{\rho_{0}} \quad u_{i}^{*}=\frac{u_{i}}{u_{0}}, \quad q^{*}=\frac{q}{q_{0}} p^{*}=\frac{p}{\rho_{0} u_{0}^{2}}$,

$V^{*}=\frac{V}{\left(V_{0}-V_{1}\right)}, E_{i}^{*}=\frac{E_{i}}{\left(V_{0}-V_{1}\right) / H}$

This leads to the following set of dimensionless parameters: $T=\frac{\varepsilon_{0} \Delta V}{\rho_{0} v K_{0}}$ which is the ratio of Coulomb and viscous forces.

$C=\frac{q_{0} H^{2}}{\varepsilon_{0} \Delta V}$ dimensionless measure of the injection level $M=\frac{1}{K_{0}}\left(\frac{\varepsilon_{0}}{\rho_{0}}\right)^{1 / 2}$ accounts for the electrohydrodynamic properties of the liquid.

$R e=\frac{\rho_{0} u_{0} H}{\mu}$ is the classical Reynolds number.

Several choices for the velocity reference $u_{0}$ are possible. In this study the most obvious choice could be $u_{0}=u_{\max }$ which is the axial inlet velocity. For convenience we shall, also use $R=\frac{T}{M^{2}}$ which is defined as the electrical Reynolds number.

If we drop the star indices for clarity, the set of nondimensional equation becomes:

$$
\begin{aligned}
& \nabla \cdot \vec{u}=0 \\
& \frac{\partial \vec{u}}{\partial t}+(\vec{u} \cdot \nabla) \vec{u}=-\nabla p+\frac{1}{R e} \Delta \vec{u}+\frac{C M^{2} R^{2}}{R e^{2}} q \vec{E} \\
& \frac{\partial q}{\partial t}+\nabla \cdot\left(q\left(\vec{u}+\frac{R}{R e} \vec{E}\right)\right)=0 \\
& \Delta V=-C q \\
& \vec{E}=-\nabla V
\end{aligned}
$$




\subsection{NUMERICAL METHOD}

The numerical procedure used to solve the entire set of coupled Navier-Stokes and EHD equations is similar to the one already addressed in previous papers [10], [11] and thus will not be discussed further more here. The boundary conditions are depicted on the Figure 2. The computations always start from the liquid at rest.

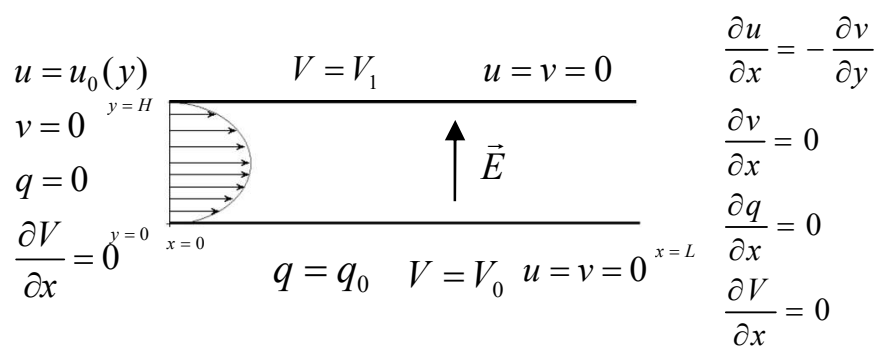

Figure 2. Boundary conditions.

In the computations that we have conducted we have considered the strong injection regime with $C=10$, the mobility parameter $M$ may be varied from 10 to 100 according different numerical experiments carried out.

\section{RESULT AND DISCUSSION}

\subsection{FLOW STRUCTURE}

In absence of charge injection i.e $T=0$ and for moderate Reynolds number the flow is steady and structured in parallel streamlines with parabolic velocity profiles in vertical sections (see Figure 3).

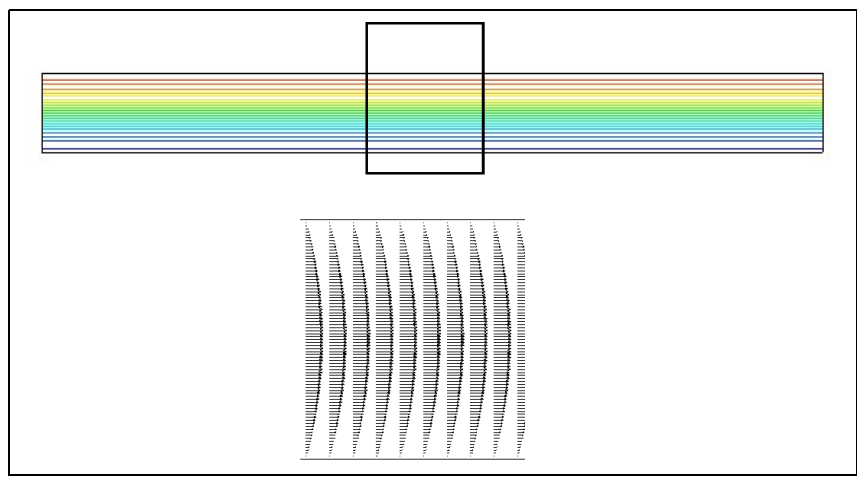

Figure 3. Flow pattern of the non-perturbated flow. Streamlines and velocity profiles in the mid-section for $R e=1$ and $T=0$.

When $T$ is increased, charge injection occurs and the ions are dragged by the vertical electric field but also by the flow. This results in a zone which is almost free of charges as it is depicted on the following figure and that Atten et al charcaterized by a so called electric entry length $\mathrm{L}_{\mathrm{E}}$ [12].

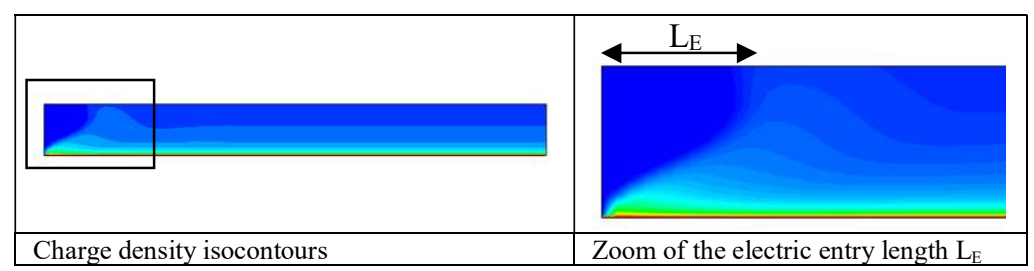

Figure 4. Snapshots of the charge density isocontours for $R e=1, T=100$ and $M=10$. Lef entire domain, right zoom of the entry zone.

Below a critical value $T_{c}$ which is linked to the Reynold number Reand ionic mobility parameter $M$, the flow is steady and a small recirculating zone appears on the top electrode (see Figure 5). This eddy is due to the presence of the electric entry length. For $R e=1$ the $T_{c}$ value is very near from the one expected for $R e=0$ which would be theoretically 164 [13].

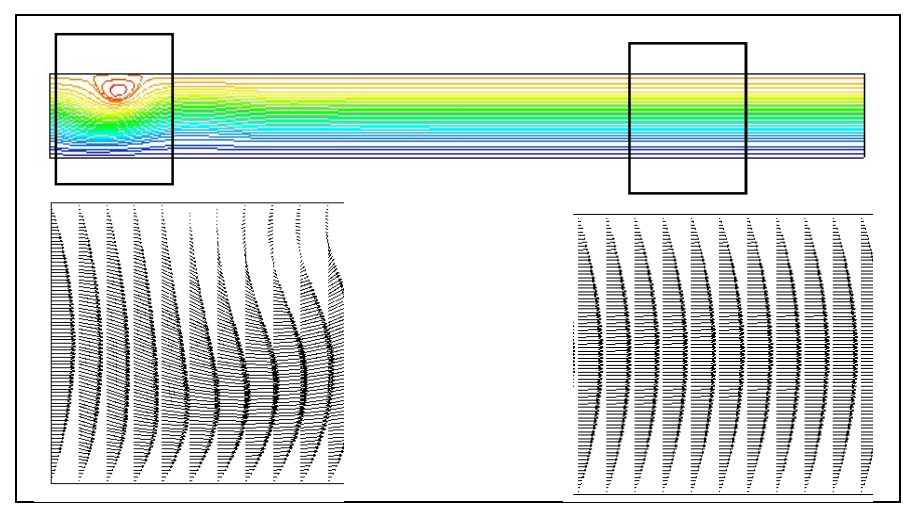

Figure 5. Streamlines and velocity profiles in two different sections for $R e=1$ and $T=100$ and $M=10$.

Downstream, after the electric entry length the charge density profile and the hydrostatic solution perfectly match as it is shown on Figure 6.

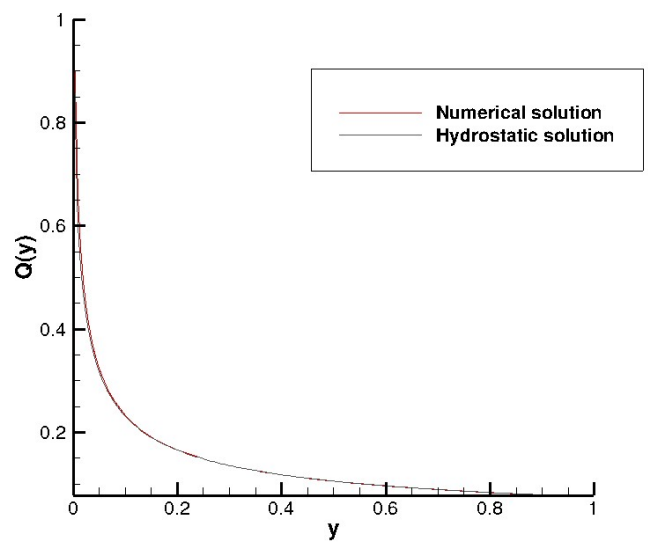

Figure 6. Comparison between the numerical and analytic hydrostatic solution of the charge density profile for $R e=1, T=100$ and $M=10$.

Above the critical value $T_{C}$ electroconvective rolls arise as in the case of an infinite long layer of fluid at rest. However 
because of the upstream flow, these electroconvective rolls are periodically convected by the main flow downstream. These roles are clearly visible on the next caption on Figure 7 where we have displayed the isocontours of the stream function for $T=200$ which is above the critical value $T_{c}$.

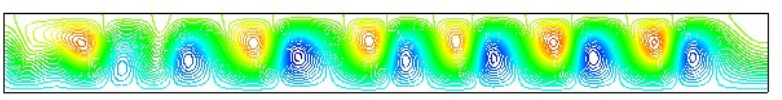

Figure 7. Instantaneous isocontours of the stream function for $\operatorname{Re}=1, T=200$ and $M=10$.

On Figure 8 we can observe the charge density isocontours at different dimensionless time $\mathrm{t}$. Three particular convective rolls are identified by letters $\mathrm{A}, \mathrm{B}$ and $\mathrm{C}$ to follow the flow motion with time.

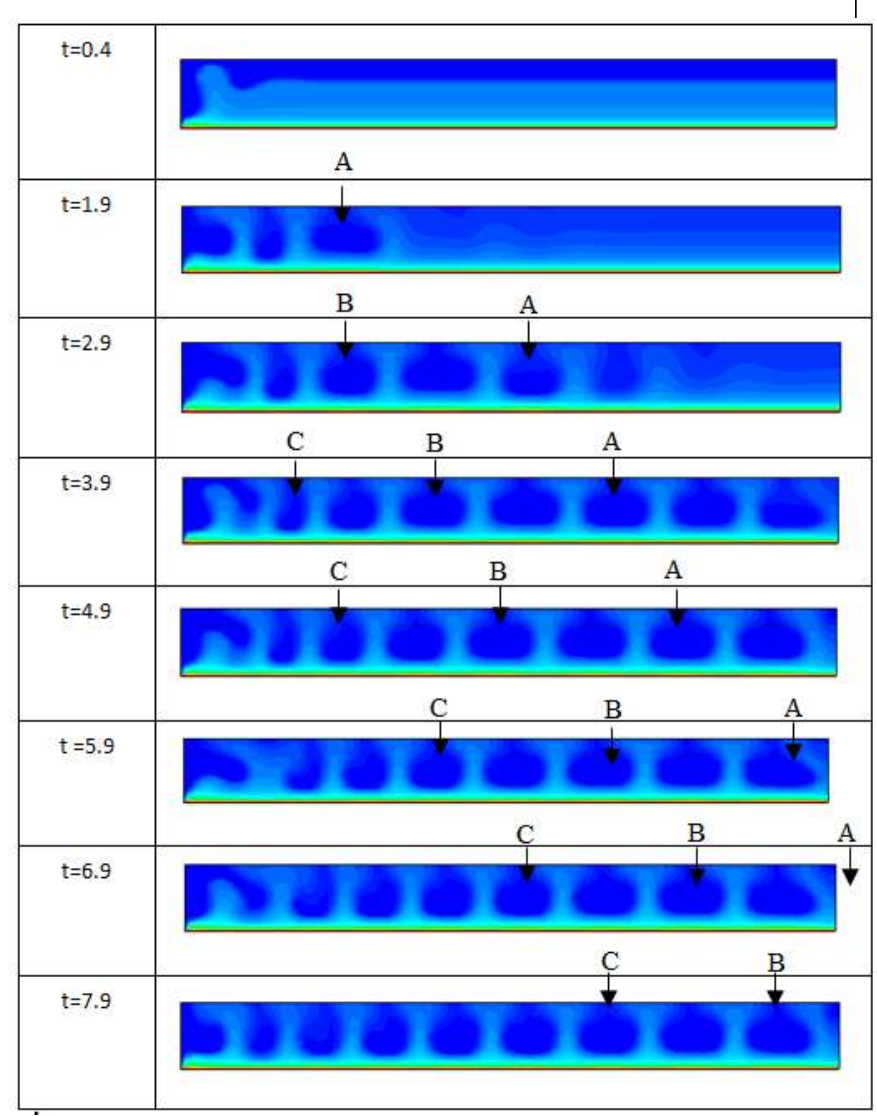

Figure 8. Time evolution of the charge density isocontours for $R e=1$, $T=200$ and $M=10$.

Figure 9 depicts the charge density distribution at the same time $\mathrm{t}=10$ for different values of $T$ in the case $M=10$ and $R e=1$. For $T=100$ the flow is steady and the electric entry length is well characterized. The electric entry length is defined as the separatrix between the part of the domain completely free of electric charges and the rest of the domain. For $T=100$ the flow is steady as it can be seen on the Figure 10 where we have reported the time history of the maximum of the vertical velocity in the whole domain. When $T$ is slightly increased the flow becomes unsteady but is still periodic. See Figure 10. When $T$ is still increased $(T=140)$ it can be seen on the same figure that the amplitude of the signal is increased and the frequency decreased. For $T=170$ we observe a significant change of the signal form which highlights a characteristic transition in the flow. Indeed for $T=170$ we can observe the appearance of electroconvective transverse rolls which explains this flow transition. For a higer $T=180$ the same type of signal is reported but its amplitude is slightly increased.

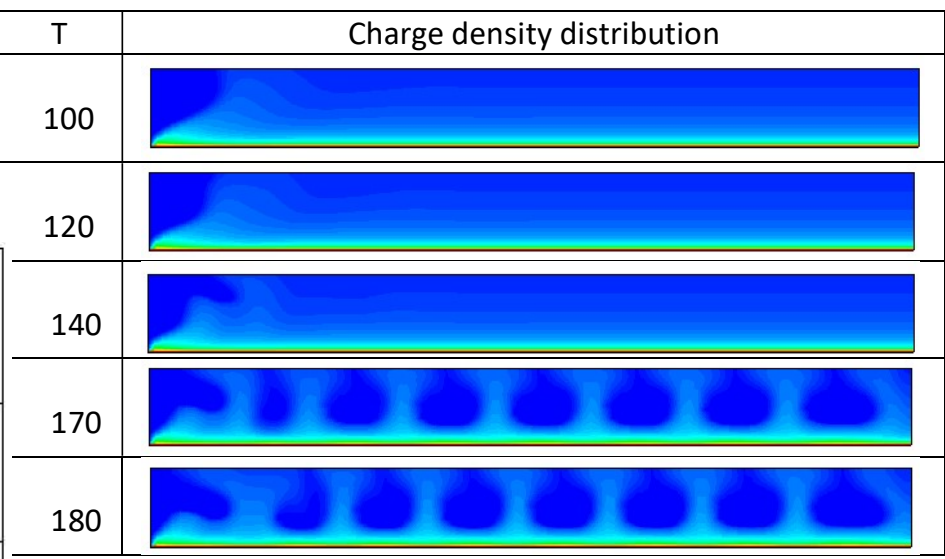

Figure 9. Charge density isocontours distribution at the same time step $\mathrm{t}=10$, for different $T$ values, for $M=10$ and $R e=1$.

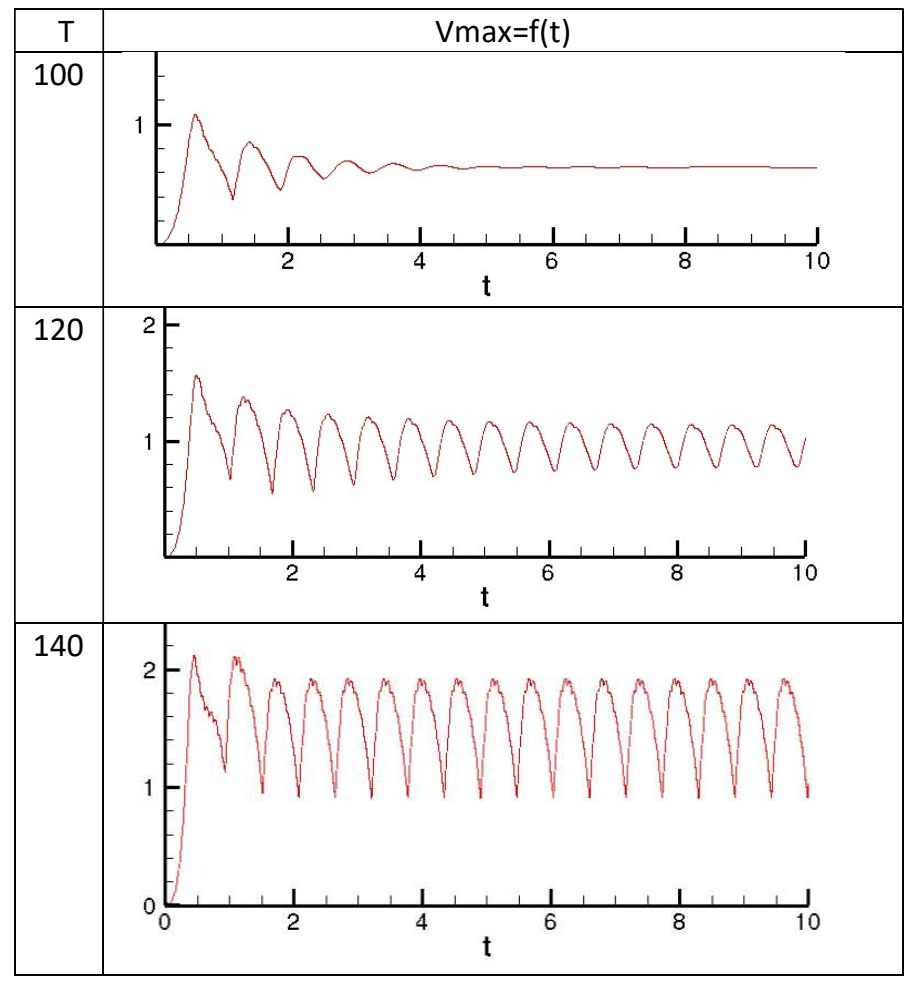




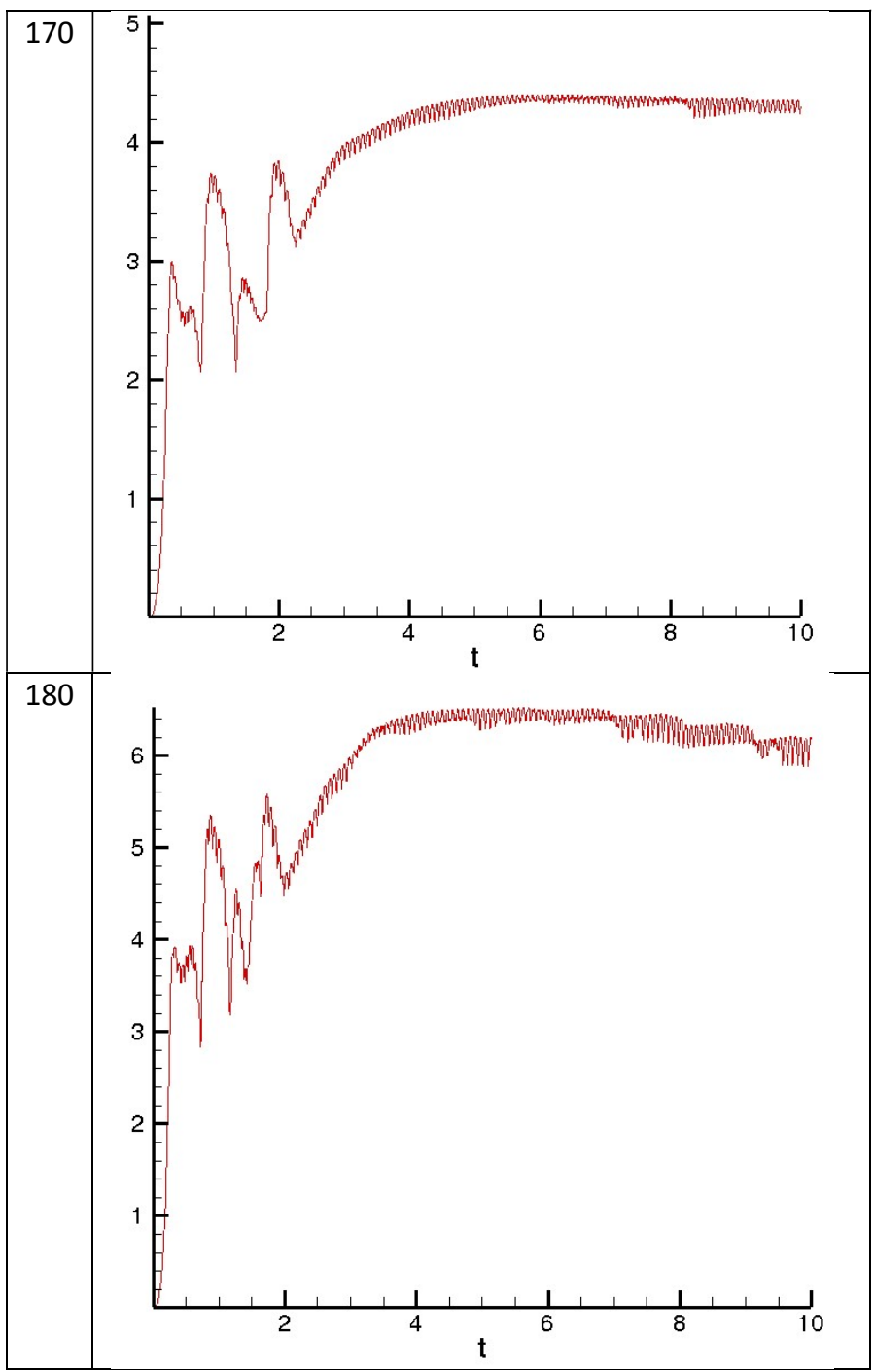

Figure 10. Time history of the maximum of the velocity Vmax in the domain, for different $\mathrm{T}$ values, for $\mathrm{M}=10$ and $\mathrm{Re}=1$.

These computations reveal that for a given Reynolds number will correspond a unique critical value of $T$ designed as $T_{c}$ for which the flow will turn from a periodic flow with slight oscillations to an unsteady one with fully developped electroconvective rolls (see the difference between cases $T=140$ and $T=170$ on Figure 9 and Figure 10). When $R e=0$ it has been found by the stability analysis that the critical $T$ parameter is $T_{c}=164$ [13]. From our computations and for $R e=1$ the critical value has been found to be between the range $[162.5,165]$. For higher values of $R e, T_{c}$ also increases.

In table 1 we have reported the critical value $T_{c}$ for different Reynolds number. For all these computations $M=10$. In electroconvective flows in closed or infinite cavities the $T_{c}$ can be numerically determined with a very high accuracy using a pseudo analytical method as in it has been shown in [15][14]. Indeed in these cases, the transition occurs between a fluid at rest and a fluid set into motion, which facilitates the determination of the critical $T$ parameter. When a Poiseuille flow is surimposed to the developpement of electroconvective intsability the transition is more fuzzy since different regimes occurs before obtaining fully developped electroconvective transverse rolls in the whole domain (see Figure 9 and Figure 10). This explains why $T_{c}$ is better expressed in a range of values.

Table 1. Critical $T$ parameter versus Reynolds number for $M=10$ and $C=10$.

\begin{tabular}{c|c|c|c|c}
\hline$R e$ & 1 & 5 & 10 & 20 \\
\hline$T_{c}$ & {$[162.5,165]$} & {$[400,425]$} & {$[500,550]$} & {$[1200,1300]$} \\
\hline
\end{tabular}

When the Reynolds number is still increased the electric charges generated at the injecting electrode are immediately swept by the flow. This requires to dramatically increase the $T$ parameter to trigger electroconvective instability in the flow and the appearance of transverse rolls. However for this high value of $T$ the flow structure changes completely and instead of purely defined electroconvective rolls we rather observe the arising of electro plumes in the bulk as it is clearly depicted in Figure 11. A new regime is therefore highlighted according the value of $T$.

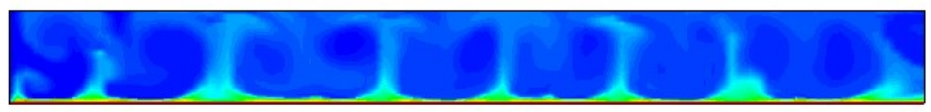

Figure 11. Snapshot of the charge density isocontours distribution at time $\mathrm{t}=6.1$ for $R e=100$ and $T=5000$ which shows the emergence of electro plumes.

\subsection{INFLUENCE OF THE IONIC MOBILITY}

The purpose of this next sub-section is to investigate the influence of the mobility parameter on the flow dynamic behaviour. In this next experiment, related to Figure 12, we have performed several computations varying the ionic mobility parameter $M$. In the next figure we have highlighted the flow behaviour through this variation of $M$ for $R e=1$ and $T=200$. For small $M$ values, the ionic mobility is large which means that transport of the electric charges carrier in the bulk is enhanced. So that electroconvective rolls can developp in the bulk. For greater $M$ values, the ionic mobility is smaller and thus the electric boundary layer is smaller and concentrated near the injecting electrode. Electroconvective cells are not able to grow. We also can observe consequently that the entry length becomes larger when $M$ is increased. It plays a role analogous to the Reynolds number. It can be observed indeed that the case $(M=10, R e=30)$, see Figure 14 is quite similar to the case $(M=60, R e=1)$ Figure 12 . So it means that the value of $T$ as well as the value of the Reynolds number are not sufficient to decide wether or not electroconvective rolls could developp in the bulk. $M$ is therefore also a key parameter for electroconvective motion and it appears that the increase of $M$ has an stabilizing effect on the flow motion. 


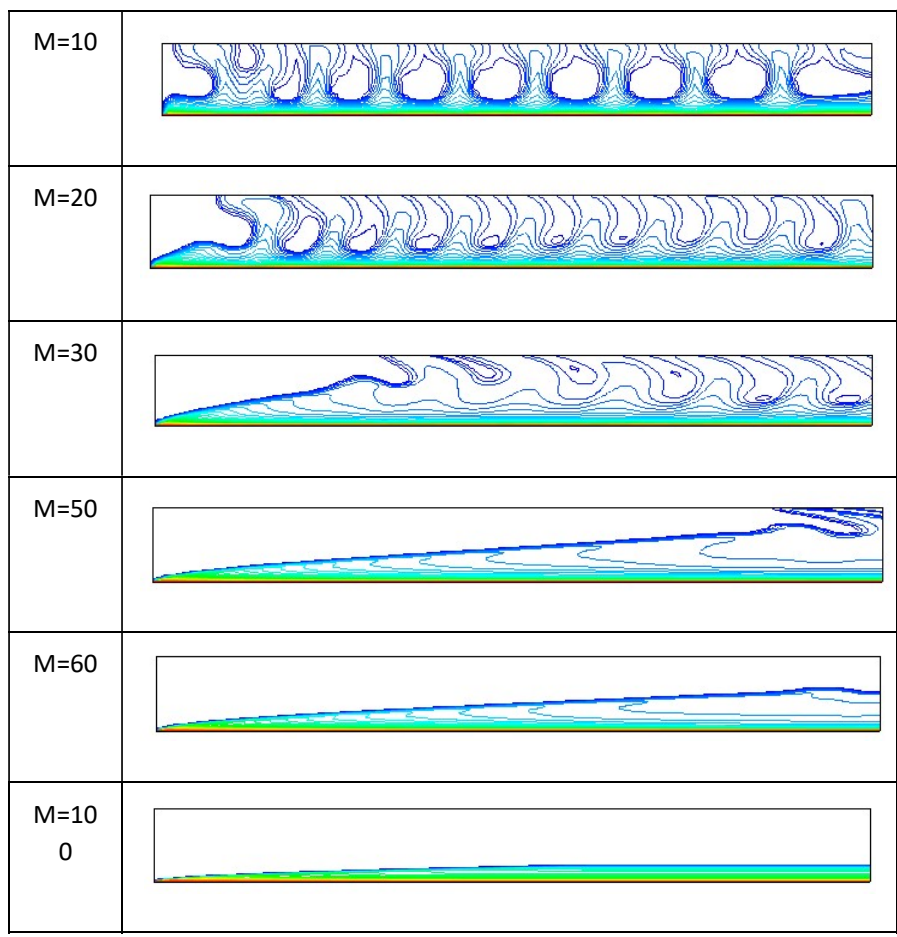

Figure 12. Snapshot of the charge density isocontours distribution at the same given time for different values of the $M$ parameter, $R e=1, T=200$

Hence from the previous results it could be expected that $T_{c}$ should increase with $M$. That's what we could notice on Figure 13 where we have depicted the instantaneous charge density distribution for different $M$ and $T$ values for the same Reynolds number $R e=1$. For $M=10$ and for $T=200$ we can observe a fully development of electroconvective rolls while for a higher $M$ values at the same $T$ the flow is steady with a very long entry length. Thus it appears that the $T$ parameter has to be increased to trigger the development of the electroconvective instability giving rise to transverse rolls. Indeed, setting $T=400$ reveals a periodic flow but the electroconvective rolls are not fully developped. We have to increase $T$ up to 800 to observe electroconvective rolls. Then it is concluded that for $R e=1$ and $M=50 T_{c}$ is in the range [700800] while for $M=10$ we found previously $T_{c}$ in the range $[162.5,165]$.

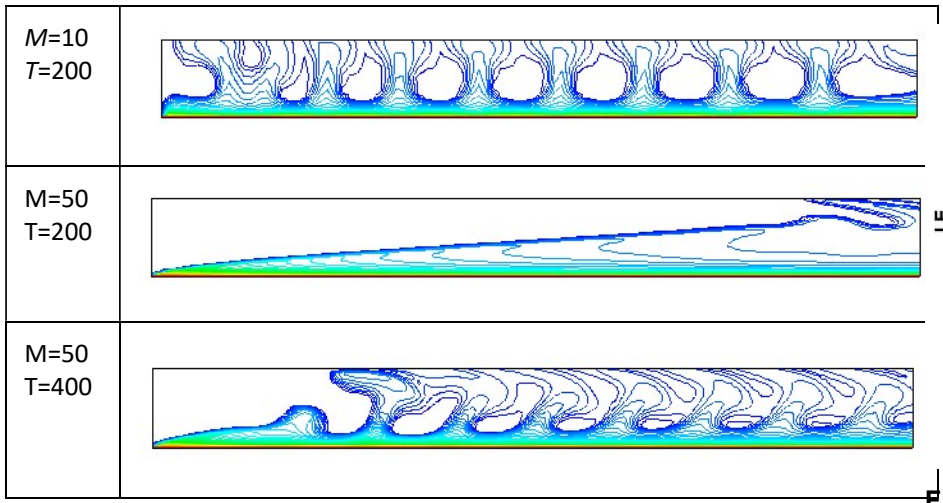

$M=50$

$\mathrm{T}=800$

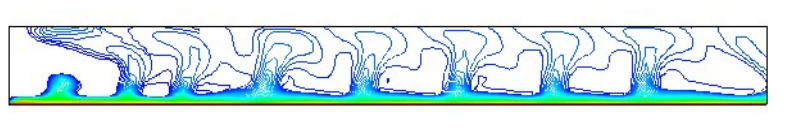

Figure 13. Comparison of the charge density isocontours distribution for different $M$ and $T$ values. $R e=1$.

\subsection{ELECTRIC ENTRY LENGTH CHARACTERIZATION}

On Figure 14 we have displayed the spatial evolution of the electric entry length characterized by the separatrix for different Reynolds numbers for the same values of $T$ and $M$. When $T$ is above the critical value for that given Reynolds number we observe a convective flow motion with electroconvective rolls (case $R e=1$ ). When we increase the Reynolds number the $T$ number becomes not large enough to trigger the appearance of the electroconvective rolls. The electric charges are only swept by the flow and the electric entry length is therefore extended.

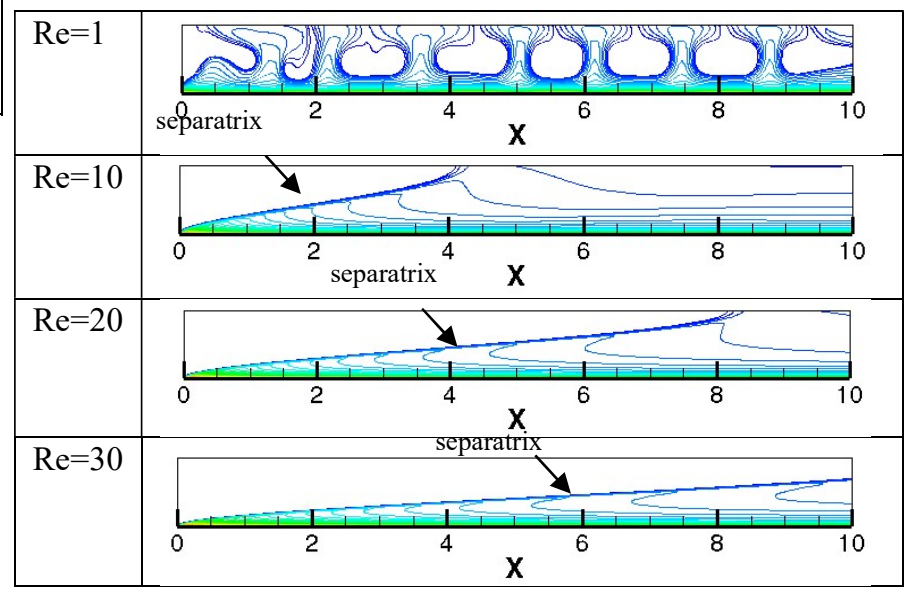

Figure 14. Evolution of the electric entry length for different Reynolds number in the case $T=200$ and $M=10$.

Figure 15 shows the linear behaviour of the electric entry length versus Reynolds number which is consistent with the result found by Atten et al [12] who expected a linear dependency.

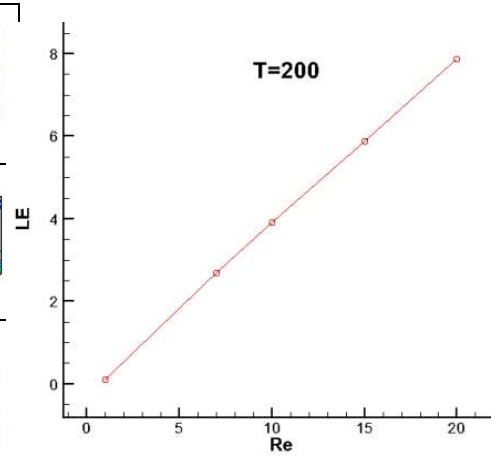

Figure 15. Electric entry length versus Reynold number for $T=200$ and $M=10$. 


\section{CONCLUSION}

Two-dimensional numerical simulations have been conducted to study the mixed convection induced by electroconvective instability in a Poiseuille flow. The flow structure has been characterized in the strong injection case $C=10$. We have highlighted the existence of electroconvective transverse rolls that are convected downstream by the flow motion when the $T$ parameter is above a critical value. It appears that the growth of these electroconvective rolls depends on the Reynolds number, on the $T$ parameter but also on the mobility parameter $M$. Great values of $M$, delay the appearance of electroconvective rolls in the domain and tend to stabilize the flow. According the Reynolds number and depending on $T$ parameter, we have emphasized different regimes passing from steady state, periodic flow, electroconvective transverse rolls and finally a regime characterized by electro-plumes for higher Reynolds and $T$ numbers. It has been pointed out that the critical parameter $\mathrm{T}_{c}$ depends on the Reynolds number and on the ionic mobility parameter $M$ as well. The electric entry length has been characterized and appears to be linearly dependent on the Reynolds number.

\section{ACKNOWLEDGMENT}

This work was partially funded by the French Government program "Investissements d'Avenir" (LABEX INTERACTIFS, reference ANR-11-LABX-0017-01) (to J. $\mathrm{Wu}$ ), and a grant of the French district Poitou-Charentes (to P. Traoré).

\section{REFERENCES}

[1] R.E. Kelly, "The onset and development of Rayleigh-Bénard convection in shear flow: a review", Physicochemical Hydrodynamics, Advance Publication, D.B. Spalding (Ed), London, UK, 1977.

[2] C. Abid and F. Papini "Thermal instabilities in a mixed convection phenomenon: nonlinear dynamics", Phys. Rev. E. Vol. 56. No. 6. pp. 6735-3744,1997.

[3] X. Nicolas, Revue bibliographique sur les écoulements de PoiseuilleRayleigh-Bénard : écoulements de convection mixte en conduites rectangulaires horizontales chauffées par le bas", Int'1. J. Thermal Sci. Vol. 41, pp. 961-1016, 2002 (in French).

[4] C.H. Yu, M.Y. Chang, T.F Lin, "Structures of moving transverse and mixed rolls in mixed convection of air in a horizontal plane channel" // Int'l. J. Heat and Mass Transfer, Vol. 40, Pp. 333-346, 1997.

[5] J.M. Luijkx, J.K. Platten and J.C. Legros, "On the existence of thermoconvective rolls transverse to a superimposed mean Poiseuille flow", Int'1. J. Heat and Mass Transfer, Vol. 24, pp. 803-817, 1981

[6] N. Agraï, Influencia de los flujos forzados sobre las inestabilidades electrohidrodinamicas, Ph.D. thesis Universidad autonoma de Madrid, Spain, 1988 (in Spanish).

[7] A. Castellanos, N. Agraït, "Unipolar injection induced instabilities in planar parallel flows", IEEE Trans. Ind. Appl., Vol. 28, pp. 513-519, 1992

[8] J.L. Lara, A. Castellanos and F. Pontiga, "Destabilization of plane Poiseuille flow of insulating liquids by unipolar charge injection", Phys. fluids, Vol. 9, No. 2, pp. 399-406, 1997.

[9] F. Martinelli, M. Quadrio and P.J. Schmid, "Stability of planar shear flow in presence of electroconvection". Turbulent Shear Flow Phenomena (TSFP), Ottawa, Canada, 2011.
[10] P. Traoré, D. Koulova-Nenova and H. Romat, "Numerical approach of the electro-thermo-convective motion in a layer of a dielectric liquid". IEEE International Conf. Dielectr. Liquid (ICDL), Coïmbra, Portugal, pp 173-176, 2005.

[11] P. Traoré, A. Pérez, D. Koulova-Nenova and H. Romat, "Numerical modelling of finite amplitude electro-thermo-convection in a dielectric liquid layer subjected to both unipolar injection and temperature gradient" J. Fluid Mechanics, Vol. 658, pp. 279-293, 2010.

[12] P. Atten and T. Honda, "The electroviscous effect ans its explanation. I the electrohydrodynamic origin: study under unipolar D.C injection", J. Electrostatic, Vol. 11, pp. 225-245, 1982.

[13] P. Atten and R. Moreau, "Stabilité electrohydrodynamique des liquids isolants soumis a une injection unipolaire", J. Mech, Vol. 11, pp. 471520, 1972 (in French).

[14] P. Traoré and A. Pérez, "Two-dimensional numerical analysis of electroconvection in a dielectric liquid subjected to strong unipolar injection" Phys. Fluids, Vol. 24, 037102; doi: 10.1063/1.3685721, 2012.

[15] J. Wu, P. Traoré, P.A. Vazquez and A. Pérez, "Onset of convection in a finite two- dimensional container due to unipolar injection of ions", Phys. Rev. E, Vol. 88, 053018, 2013.

\section{TECHNICAL BIOGRAPHIES}

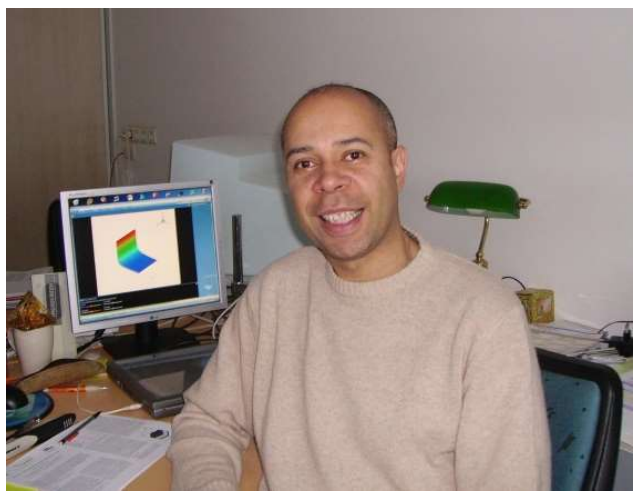

Philippe Traoré was born in Grenoble (France) in September 1963. He has been graduated from the Superior National Engineer School of Aeronautical Constructions in 1987 and received his Phd degree in fluid mechanic in 1996. $\mathrm{He}$ is currently the deputy director of the mechanical department of the University of Poitiers (France) where he teaches fluid mechanic and scientific computation. His research interests focus on general Computational Fluid Dynamic, two-phase flows, granular media as well as on ElectroHydroDynamic.

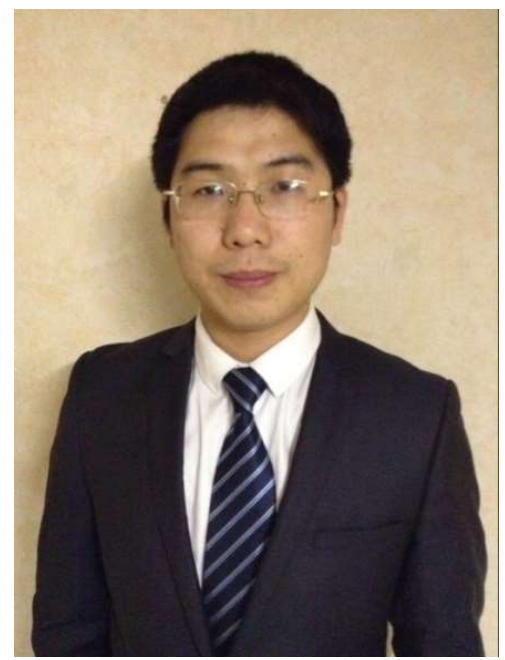


Jian Wu (M '13) was born in Jiangxi, China, in 1985. He received the B.Eng. (2006) degree in thermal energy and power engineering, the B.B.A. (2006) in business administration and the M.Eng. (2008) degree in refrigeration and cryogenic engineering from Harbin Institute of Technology, Harbin, China He received the $\mathrm{PhD}$ degree in fluid mechanics from the Electro-Fluid Dynamics Group, University of Poitiers, Poitiers, France, in 2012. He was a research engineer at University of Poitiers from Oct. 2012 to Oct. 2014. Currently he is a visiting researcher of University of Seville, Spain. His areas of research include Electro-hydrodynamics, Electro-thermo-hydrodynamics and computational fluid dynamics. He is a member of the IEEE Dielectrics and Electrical Insulation Society and the IEEE Industry Applications Society.

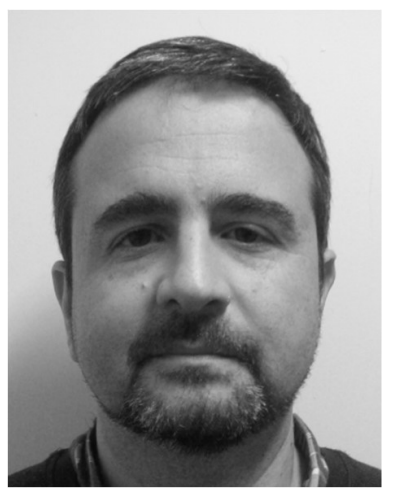

Pedro A. Vázquez was born in Seville, Spain in 1969. He received his B.Sc degree from the University of Seville, Spain in 1992, and the Ph.D. degree in Physics from the same University in 1998. He is currently Associate Professor at the Departement of Applied Physics III of the University of Seville.

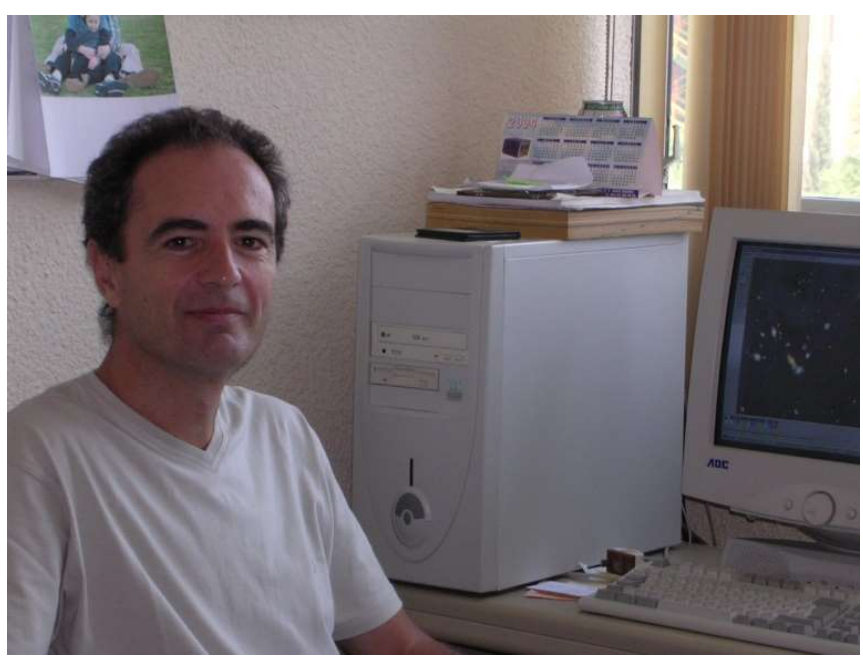

Alberto T. Pérez was born in El Puerto de Santa María Cádiz, (Spain) in 1962. He obtained a Bachelor degree from the University of Seville (Spain) in 1985 and a PhD from the same university in 1989 . He is currently Profesor at the University of Seville. He has co authored more than 40 papers on Electrohydrodynamics, Cohesive Granular Materials and Suspensions in Dielectric Liquids. He was Invited Professor at the Universities of Poitiers (2007 and 2013) and Nice (2008), both in France, and Invited Researcher at the LPMC-CNRS in Nice (2003). He served as Vice Dean of the Faculty of Physics at the University of Seville for 12 years. Dr. Pérez is member of the American Physical Society. 